\title{
12 Big Bangs
}

We are now better equipped to revisit issues related to the Big Bang (69). Is the Big Bang, as was long believed, a one-off phenomenon, or can it occur again at any time and at any place, here, now, anywhere, anywhen? Can it happen right here, right now, in your home or office, either in staggered space-time or in a present that will instantly split from ours to become an infinite future? Can it be the case that, as the cosmologist Sean Carroll once put it at a meeting of the American Astronomical Society, in a remark that was later lampooned, "a universe could form inside this room and we'd never know" (70)?

Unless we discover convincing grounds to the contrary, there is little reason to believe that there is anything unique or special about the one Big Bang we are aware of. A Big Bang can well happen in a parallel universe, either elsewhere in space and far beyond our ability to ever perceive, or in a very close interwoven universe staggered within the discrete texture of the space we find ourselves in, in such a way that no physical bond or interaction exists or occurs between the two.

Big Bangs can in principle be caused by widely different mechanisms. Roger Penrose and many others have described how Big Bangs can occur under a variety of scenarios. We will be looking in Chapter 14 at some of the other ways universes can pop into existence, and how these possible birth mechanisms impact time within the newly-born universes. In the meantime, to illustrate how readily Big Bangs can happen, let's look at an alternative scenario: how a universe can be born of a random quantum quirk - some quantum fluctuation.

If Big Bangs can come about from a quantum fluctuation from within a matrix universe, what is the statistical law governing the frequency of occurrences of Big Bangs from within one given matrix universe?

To be able to decay, an atom within radioactive element must borrow energy from nearby virtual particles, and therefore rudimentary statistics of some of the attributes of virtual particles could, within at least a certain range, be inferred from the half-life values of different radioactive elements of different molecular weights and the corresponding different levels of energy that need to be borrowed from the quantum vacuum to bring about decay. From this, we could conceivably extrapolate a rough approximation of the theoretical frequency of the emergence of outlier virtual particles, possessing attributes which may lead to a quantum fluctuation powerful enough to give rise to a Big Bang.

If a Big Bang could statistically occur, say, every 10 billion years, a new one would be long overdue within our own universe and we could expect a new one, soon to happen in some living room, or near Betelgueuse or Sirius or elsewhere. A good place to start is to understand the many ways whereby Big Bangs can occur in the first place.

Another approach was taken by Sean Carroll and Jennifer Chen at the Enrico Fermi Institute at the University of Chicago, who made a calculation of possible Big Bang occurrence statistics based on a scenario of a Big Bang arising out of quantum fluctuations of vacuum energy. To

\section{(cc) BY-NC-ND}


estimate the requisite boundary data values however, Sean Carroll and Jennifer Chen estimated a vacuum energy per an arbitrary volume unit deduced from the observed acceleration rate of the universe - and then calculated the probability that a fluctuation would be large enough to trigger a phenomenon of space-time inflation. The result of their calculation was exceedingly small but non-zero: in other words, they found a mathematically non-zero, albeit small, probability of a Big Bang happening.

In the putative 'matrix universe' whence a quantum fluctuation may have given rise to our universe, 14-odd billion years ago, the laws of physics were not necessarily the same as those we now know. They, or at least the certain values of their associated constants (71), may have been different. But under the current laws of physics, there is nothing extraordinary about a Big Bang happening from a quantum fluctuation, thereby creating a new universe.

Recapping where we are:

1. The traditional view until recently was that the Big Bang emerged from nothing from an environment where there was no environment, no matter, no time, and no laws of physics at all, and everything - including the laws of physics themselves - were born with the Big Bang.

It is however very likely not the case that absolute nothingness was ever possible, let alone is or will ever be:

"Whether there can be ever be absolute nothingness" is really two separate questions, as follows:

1a- First, can there ever have been absolute nothingness, i.e. if there had never been a universe the way we know it to begin with, and

1b- Given that something now exists, or equivalently has existed at some point, can it ever be that full nothingness can re-establish itself at some point.

Tackling question number $1 \mathrm{~b}$ first - there is no credible mechanism for full dissipation of what already is (at any arbitrary point in time when something happens to be). The only way to reach full nothingness would be to dilute everything to a fully vanishing point, but then because of infinitesimal residuals all the conditions needed to create at least one element term of the Heisenberg equation would be there - thus immediately giving rise to quantum foam, which would, by definition, not be pure nothingness. It's too late for pure nothingness to ever exist.

Which leads us to the first question (1a): Could there ever have been full nothingness in the past (which would demonstrate that time had a beginning; if there ever was absolute nothingness, then time cannot stretch backwards to infinity.)

Martin Bojowald et al. theorized that time, far from being created alongside space at a Big Bang, must precede a Big Bang for a Big Bang to be able to happen. We will discover in the next Chapter further inescapable pre-conditions for a Big Bang to occur.

This leaves open two possibilities for absolute nothingness: either math is not of universal applicability, and the equations that mandate the emergence of some material reality are not 
valid mathematical equations in circumstances other than ours or in other universes, or, any Ur-universe - the first universe ever to exist, be it our universe or any other - could not have emerged via a Big Bang.

But upon examining, this latter condition does not hold and it is relatively easy to dismiss: preconditions can also be formulated for every conceivable non-Big Bang universe birth scenario. Which leaves us with the former condition, which can be equivalently reformulated as: for absolute nothingness to ever have existed, the universe must not be a mathematical structure.

But if the universe is not a mathematical structure, then how could it pop into existence from nothingness? There seems to be no credible mechanism for an Aristotelian universe to come into being from nothingness (72). A conclusion would then inescapably have to be that the universe has always existed in some form (that form could be anything, which in turn seems to gainsay the assumption that absolute nothingness ever existed.)

2. Or, Big Bang can possibly happen under laws of physics we do not know.

3. It is possible that the laws of physics, if any, that were prevalent before or at the onset of our Big Bang, 14 billion years ago, were more or less the same as we know today (perhaps down to the value of some fundamental constants. A Big Bang can happen under our current laws of physics, and indeed, under a variety of different scenarios calling upon different laws.

4. There exist other possible scenarios for the births of individual universes, such as bouncing universes, and so forth (73).

In all cases, the bottom line is always that a new universe will suddenly Big-Bang into existence because if it did not, some mathematical law would be broken.

In other words, we will know how to create a universe if we find a mathematical equation that would be violated if the universe did not form: It's really as simple as that. The alternative would be that some form of the universe has always existed, and that time itself stretches infinitely backwards.

Here's how a universe can pop into being from nothing - just so that an applicable mathematical equation not be violated.

First, let's recall that time and energy, or equivalently to energy, mass (under Einstein's equation $\mathrm{e}=\mathrm{mc}^{2}$ ) are 'orthogonal' (as defined earlier.)

Combined with the discreteness of time, the effects of the orthogonality of time and mass can create universes out of nothing. The only requirement is that the matrix space-time begetting the new universe is already discrete, at least within the area whence the new universe arises.

In the quantum foam of so-called virtual objects and particles, the virtual objects that fleetingly inhabit it always stay 'under the radar' of perceivability and hence do not become part of our manifest, tangible, stable reality. This 'quantum vacuum' is a froth of seething virtuality where fleeting, ghostlike things continuously pop in and out of almost-existence. These things do not manifestly exist: hence the term 'virtual' as in virtual particles, virtual objects, virtual dimensions, even virtual time, etc., and 
remain in their condition of virtual existence just briefly enough to not become real. However, crucially, they impact, or interfere with, the real world in a myriad of ways, sometimes even lending ghostlike properties to the real world, such as in the tunnel effect (74).

Energy can be borrowed, although for a very short time only, from the virtual objects that make up the vacuum's quantum foam. The statistical distribution of the energy levels available from virtual objects is not known. What is certain, however, is that on occasion spikes of high virtual energy can happen - any level of energy is in principle allowed, provided that the corresponding duration of existence of that energy remain under its authorized time threshold. In essence, the more energetic a virtual object, the less time it can exist.

Let's imagine that a particularly rare event occurs: a humongously big virtual object pops up within the quantum foam.

To create a universe out of the immaterial quantum foam, all we would need to do would be to make this virtual, rare, massive object manifest (i.e. make it instantly real) to wrest it, as it were, from under the cloak of invisibility and immateriality of its virtual existence. If we manage that, we will create a Big Bang, which would be nothing else than this massive virtual object instantly becoming real.

How can this happen? As it turns out, rather easily. Energy and time are orthogonal: When virtual things (particles, exotic objects, energy embodied as a virtual mass, etc...) appear, they may linger on about in the unreal limbo of their virtual existence for a duration of time only at most as long as Heisenberg's equation permits it.

Nothing in principle prevents a huge virtual mass from popping into virtual existence, even if it only happens very seldom. So let's imagine that once in a very long while a very massive virtual object, $M$, does pop into virtual existence - a mass which happens to be so huge that the maximum allowed span of time it is permitted by Heisenberg's equation to remain in virtual existence happens to be smaller than the then-valid quantum of time.

But $\mathrm{M}$ cannot remain in existence for a duration less than the quantum of time, because this quantum of time is incompressible.

Nor will reality allow a valid mathematical equation to be violated, which however looks exactly like it is about to happen here, because the maximum duration allowed for the virtual life of $\mathrm{M}$ by Heisenberg's equation turns out to be shorter than the quantum of time.

Put equivalently, the duration of time that would permit Heisenberg's equation to function properly would be smaller than the incompressible reigning quantum of time. In other words, a mathematical law is going to be violated.

This cannot happen.

The only possible way violation can be averted is if the mass becomes instantly real:

\section{BANG}


How actual reality takes over from that point on - with an inflation phase, say, or any other way - is out of the hands of the birthing mechanism. The mass is now in another world entirely than the ghostly virtual world whence it emerged. It has now actualized itself into manifest reality. Physical reality, with its laws and constraints, its sweat and tears, is now taking over.

This scenario is, by the way, not entirely neutral or transparent in terms of the virtual quantum foam it leaves behind. It is likely that it creates with it a trail of other, smaller universes, most of which will probably soon dissolve back into the vacuum, creating within it large-scale disturbances in the foam and frothing it up for a while. It also requires that the space time within the prior matrix universe be discrete, and rediscovers via a very different route and within the narrow case of a quantum fluctuation, Martin Bojowald's more general requirement of time itself existing prior to a Big Bang. 